\title{
Coristoma ósseo mesentérico em uma porca
}

\author{
Mesenteric Osseous Choristoma in a Sow \\ Karine Ludwig Takeuti', Gabriela Fredo ${ }^{2}$, Rafael Cé Viott', David Driemeier ${ }^{2}$ \\ \& David Emilio Santos Neves de Barcellos ${ }^{1}$
}

\begin{abstract}
Background: Osseous choristomas represent the production of osseous tissue in abnormal regions, such as subcutaneous, fibrous or perivascular tissues, skeletal muscle and skin. These structures have been found in humans, dogs (lungs and dura mater), horses (large intestines) and cattle (lungs and mesenteric lymph nodes). They were also found in the mesenteric region and thoracic cavity of sows and piglets. The aim of this paper was to describe the macro and microscopic lesions found in a sow which died suddenly by a rare mesenteric osseous choristoma.

Case: A lactating sow, parity four, from the Swine Department of the Federal University of Rio Grande do Sul (UFRGS), Brazil, showed appetite loss, hyperthermia $\left(41.5^{\circ} \mathrm{C}\right)$ and sudden death. The animal was submitted to necropsy at the Department of Veterinary Pathology, UFRGS. On external examination, the sow showed conjunctival and perioral cyanosis, proximal colon region with a firm structure with approximately $3.0 \mathrm{~cm}$ length in mesenteric area. Moreover, the colon was ruptured and fibrin deposition and intestinal content in abdominal cavity were observed. Fragments of organs were collected in $10 \%$ formalin, routinely processed for histology and colored with hematoxylin and eosin stain (H\&E). Histologically, it was found bone trabeculae with osteoid, periosteum, spaces with osteocids and endochondral ossification with bone marrow composed by adipose tissue and intense proliferation of conjunctive stroma. The mesenteric lymph nodes showed intense lymphoid hyperplasia with multifocal centrilobular necrosis. There was fibrin in serosa of colon, acute peri-spleen and acute fibrinopurulent perihepatitis.

Discussion: Macroscopic and microscopic findings were consistent with mesenteric osseous choristoma. This condition is unusual in domestic animals, however it has been already described in pigs. Osseous choristoma could be related to previous mesenteric torsions, which could occur in growing phase, causing inflammation and hemorrhage, stimulating its generation. These structures may cause lesions in adjacent organs. The abrupt movements of the sow or intestinal peristalsis could have broken the bone, raising two tips, which in contact with the colon, ruptured it. Because of this, shedding of intestinal content was observed, which resulted in an acute peritonitis and sudden death of the animal. The annual mortality rates of sows (natural deaths or euthanasia) varies from 4.0 to $6.0 \%$, but may be variable. Sow sudden deaths represent $17.5 \%$ of all sows and boars deaths, and gilts, pregnant and lactating sows are more susceptible. The most common causes of sow sudden death are gastrointestinal problems, such as ulcer, enteritis, torsions and ruptures; pneumonia; urinary infections, such as cystitis and pyelonephritis; heart failure; dystocia, and septicemia. However, one third of sudden death causes are not identified. The necropsy is the only way to identify the cause of death, through observation of macroscopic lesions and subsequent laboratory tests. Better knowledge of causes is important to establish control measures and possibly reduce culling of swine females. The causes of sudden death in sows should be better understood. Case reports of mesenteric osseous choristomas are rare in pigs, however it is not possible to assert if its occurrence is low, since few sudden deaths in sows are investigated.
\end{abstract}

Keywords: sudden death, heterotropic bone, metaplasic bone, swine.

Descritores: morte súbita, osso heterotrópico, metaplasia óssea, suínos. 


\section{INTRODUÇÃO}

Coristomas ósseos ou metaplasias ósseas consistem na produção de tecido ósseo histologicamente normal em locais anormais $[3,4,10]$. A patogênese da formação de ossos ectópicos depende de quatro fatores principais [3]: (1) necessidade de algum fator desencadeante, por exemplo um trauma local; (2) resposta tecidual; (3) presença de células mesenquimais, que na presença do sinal tecidual se diferenciam em osteoblastos ou condroblastos; (4) presença de um ambiente que permita a formação do osso ectópico continuadamente. Essas formações ósseas podem ser classificadas em dois tipos: heterotópicos, quando as células são normais, porém presentes em locais incomuns; ou formados por metaplasia óssea, cuja origem é um outro tipo de tecido conjuntivo, por exemplo, fibroblastos que se diferenciam em osteoblastos [4].

Embora sejam raros, coristomas ósseos podem ser encontrados em tecidos subcutâneo, fibroso e perivascular, musculatura esquelética e pele [3]. Em humanos há relatos envolvendo formações ósseas em locais incomuns após traumas pequenos e repetidos em um mesmo local [9]. Já em animais domésticos foram observadas em pulmões e na dura mater de cães [4], em equinos associadas a adenocarcinoma de intestino grosso [2], e em pulmões [4] e linfonodos mesentéricos de bovinos [6]. Estas estruturas também foram encontradas na região mesentérica de porcas $[10,12]$ e na cavidade torácica de leitões [7].

O objetivo desse trabalho foi relatar um caso de coristoma ósseo no mesentério de uma fêmea suína.

\section{CASO}

Uma porca lactante de ordem de parto quatro do Setor de Suínos da Universidade Federal do Rio Grande do Sul (UFRGS) apresentou hiporexia e hipertermia $\left(41,5^{\circ} \mathrm{C}\right)$. Após morte súbita, o animal foi encaminhado ao Setor de Patologia Veterinária (SPV) da UFRGS para necropsia. Ao exame externo, a porca apresentava cianose das mucosas conjuntivais e oral. $\mathrm{Na}$ abertura da cavidade abdominal, observou-se no mesentério em região do cólon proximal, área de coloração branca e consistência rígida medindo aproximadamente $3,0 \mathrm{~cm}$ com espessura de 1,0 cm (Figura 1A). Além de área de perfuração do cólon com deposição de fibrina e conteúdo intestinal livre na cavidade

Durante a necropsia, fragmentos de diversos órgãos foram coletados e armazenados em solução de formalina $10 \%$ e processados rotineiramente para exame histopatológico e corados com hematoxilina-eosina (H\&E).

Histologicamente foi observada no mesentério a presença de trabéculas ósseas com osteóide, periósteo, lacunas com osteócitos e ossificação endocondral com medula óssea constituída de tecido adiposo, associado a intensa proliferação de denso estroma conjuntivo adjacente a este crescimento (Figuras 1B, 1C e 1D). O linfonodo mesentérico apresentava intensa hiperplasia linfoide com necrose centrolobular multifocal moderado. No cólon havia a presença de restos de fibrina na serosa do cólon. O baço apresentava necrose fibrinoide e presença de bactérias na serosa e fibras vegetais, compatível com uma periesplenite aguda, e o fígado apresentava peri-hepatite fibrinopurulenta aguda.

\section{DISCUSSÃO}

Os achados macroscópicos e histológicos foram compatíveis com um coristoma ósseo no mesentério do animal. Coristomas ósseos são incomuns em animais domésticos, porém sua presença na região mesentérica de suínos já foi relatada [10,12]. Acredita-se que a causa de ossificações ectópicas em suínos esteja relacionada a torções de mesentério parciais prévias [12] ocasionadas ainda na fase de crescimento, resultando em inflamação e hemorragia, estimulando sua formação. Embora não tenham importância pato-

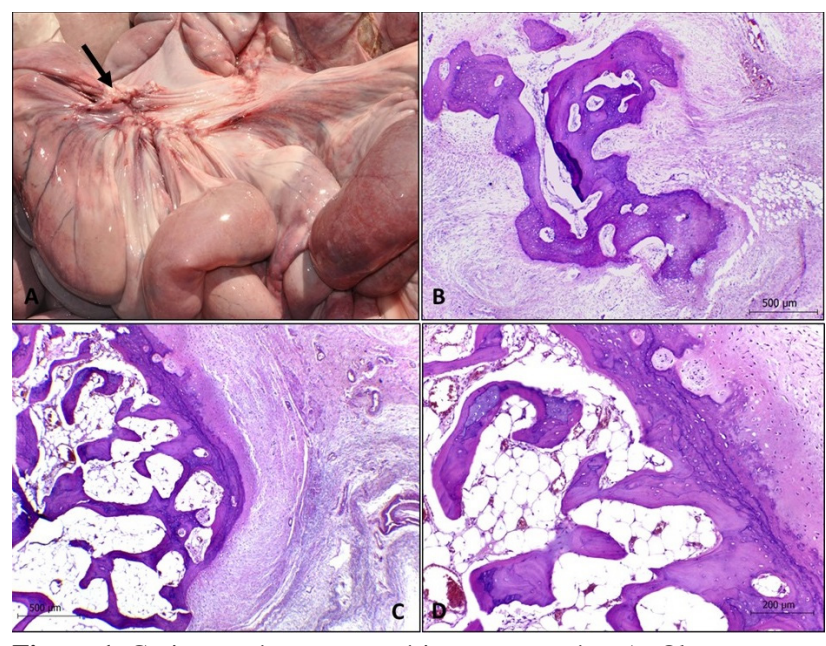

Figura 1. Coristoma ósseo mesentérico em um suíno. A- Observa-se em mesentério em região do cólon proximal, área de coloração branca medindo aproximadamente $3,0 \mathrm{~cm}$ (seta). B- Formação de matriz osteoide com intensa proliferação de tecido conjuntivo fibroso denso (Obj. 4X. H\&E). C- Observa-se a presença de trabéculas ósseas com osteoide, periósteo, lacunas com osteócitos e ossificação endocondral com medula óssea constituída de tecido adiposo, associado a intensa proliferação de denso estroma conjuntivo adjacente a este crescimento (Obj. 4X. H\&E). D- Proliferação de matriz osteoide com fibrose em canais medulares. Há ainda proliferação de tecido conjuntivo denso (Obj. 100X. H\&E). 
lógica [7], estas estruturas podem causar lesões em vísceras adjacentes. Observou-se que o osso presente no mesentério era delgado e consequentemente frágil. Os movimentos bruscos da fêmea ou o peristaltismo intestinal podem ter contribuído para a estrutura ter se partido ao meio. Dessa forma, foram originadas duas extremidades em formato de bisel, que em contato com o cólon, causaram sua ruptura e o extravasamento de conteúdo intestinal para a cavidade abdominal, iniciando-se um quadro de peritonite aguda que resultou na morte do animal.

As taxas de mortalidade anuais normais (morte natural ou eutanásia) de matrizes suínas são de 4-6\% [1], no entanto esses valores podem ser muito variáveis. Mortes súbitas de porcas representam $17,5 \%$ do total de mortes de suínos adultos, sendo que fêmeas mais jovens e em gestação ou lactação são mais suscetíveis [8]. Dentre as causas de morte súbita de matrizes su- ínas, destacam-se problemas gastrintestinais (úlceras, enterites, torções, rupturas), pneumonias, infecções urinárias (cistites, pielonefrites), falhas cardíacas, distocias e septicemias $[5,8,11]$. No entanto, cerca de um terço das causas de morte súbita não são identificadas [8]. Somente após a realização da necropsia foi possível diagnosticar a causa da morte do animal, evidenciando a importância desta prática para um correto diagnóstico e melhor conhecimento das causas de morte súbita em porcas, que são pouco estudadas. Relatos de ossificação mesentérica em suínos são escassos, porém não é possível afirmar que sua ocorrência seja baixa, já que nem todas as causas de morte súbita de porcas são investigadas, sendo inviável a identificação da causa mortis desses animais.

Declaration of interest. The authors report no conflicts of interest. The authors alone are responsible for the content and writing of the paper.

\section{REFERENCES}

1 Almeida M.N., Lippke R.T., Vearick G., Mellagi A.P.G., Bortolozzo F.P., Wentz I. \& Barcellos D.E.S.N. 2007. Aspectos epidemiológicos e controle da mortalidade de matrizes na suinocultura tecnificada. Acta Scientiae Veterinariae. 35: 91-99.

2 Kirchhof N., Steinhauer D. \& Fey K. 1996. Equine adenocarcinomas of the large intestine with osseous metaplasia. Journal of Comparative Pathology. 114: 451-456.

3 McCarthy E.F. \& Sundaram M. 2005. Heterotopic ossification: a review. Skeletal Radiology. 34: 609-619.

4 Meyer R.K. \& McGavin M.D. 2007. Cellular and Tissue Responses to Injury. In: McGavin M.D. \& Zachary J.F. (Eds). Pathologic Basis of Veterinary Disease. 4th edn. St. Louis: Mosby Elsevier, pp.36-62.

5 Morés N. \& Morés M.A.Z. 2012. Morte de matrizes em granjas de suínos. In: Sobestiansky J. \& Barcellos D.E.S.N. (Eds). Doenças dos Suínos. 2,ed. Goiânia: Cânone Editorial, pp.778-782.

6 Ohfuji S. 2012. Osseous metaplasia in the mesenteric lymph node with metastatic cholangiocarcinoma in a cow. American Journal of Cancer Science. 1(1): 1-7.

7 Palmer N. 1993. Bones and Joints. In: Jubb J.V.F., Kennedy P.C. \& Palmer N.C. (Eds). Pathology of Domestic Animals. 4th ed. Toronto: Academic Press, pp.1-181.

8 Palomo A. 2006. Analysis of sow mortality among breeding sows in Spanish pig herds. In: Proceedings of the Allen D. Leman Conference (Saint Paul, U.S.A.). p.30.

9 Puzas J.E., Miller M.D. \& Rosier R.N. 1989. Pathologic bone formation. Clinical Orthopaedics Related Research. 245: 270-281.

10 Sanford S.E. \& Rehmtulla A. 1994. Heterotopic mesenteric bone: an unexpected cause of mesenteric torsion in a sow. Swine Health and Production. 2(1): 17-18.

11 Stalder K., D'Allaire S., Drolet R. \& Abell C. 2012. Longevity in Breeding Animals. In: Zimmermann J.J., Karriker L.A., Ramirez A., Schwartz K.J. \& Stevenson G.W. (Eds). Diseases of Swine. 10th edn. Ames: Wiley-Blackwell, pp.50-59. 12 Thomson J.R. \& Friendship R.M. 2012. Digestive System. In: Zimmermann J.J., Karriker L.A., Ramirez A., Schwartz K.J. \& Stevenson G.W. (Eds). Diseases of Swine. 10th edn. Ames: Wiley-Blackwell, pp.199-226. 